\title{
CONCENTRACIÓN MAGNÉTICA APLICADA A RELAVES DE COBRE
}

\author{
L. VALDERRAMA ${ }^{1 *}$; M. SANTANDER $^{1}$; B. ZAZZALI ${ }^{1}$ y M. CARMONA ${ }^{2}$ \\ ${ }^{1}$ Universidad de Atacama \\ ${ }^{2}$ Empresa Nacional de Minería \\ luis.valderrama@uda.cl
}

Artículo recibido septiembre/2014 y aceptó en diciembre/2014

DOI: $10.15628 /$ holos.2014.2623

\section{RESUMO}

Este trabajo tiene como objetivo desarrollar un circuito de concentración magnética de baja intensidad para tratar relaves producidos en el tratamiento de minerales de cobre. Para este estudio fueron recolectadas muestras de 27 calicatas construidas y distribuidas a lo largo de la corona del tranque de relaves de la Planta Manuel Antonio Matta de la Empresa Nacional de Minería. El análisis mineralógico indicó la presencia de cuarzo, ortoclasa, magnetita, actinolita, biotita, clinocloro ferroso, albita cálcica, calcita, hematita, pirita, pirrotina y calcopirita. El análisis químico mostró un contenido de hierro total de $18,5 \%$ y de hierro magnético de $15,2 \%$. Pruebas realizadas en un Tubo
Davis indican que se puede obtener un concentrado con ley de $66,4 \%$ de hierro, 3,9\% $\mathrm{SiO}_{2}, 0,70 \% \mathrm{Al}_{2} \mathrm{O}_{3}, 0,69 \%$ de $\mathrm{MgO}, 0,60 \%$ de $\mathrm{CaO}$ y 0,178 de azufre, siendo la recuperación de hierro del $79,2 \%$. Se realizaron pruebas de concentración, a escala de laboratorio, en un separador magnético WLIMS. El concentrado rougher del separador magnético fue remolido hasta una granulometría cuyo $\mathrm{P}_{80}$ es de $50 \mu \mathrm{m}$, siguiendo luego pruebas de limpieza, que entregaron un producto final con una ley de hierro de $63,1 \%$, hierro magnético 61,83 $\%$, azufre $0,23 \%, \mathrm{SiO}_{2} 7 \%, \mathrm{Al}_{2} \mathrm{O}_{3} 1,16 \%, \mathrm{CaO} 0,52 \%$ y $\mathrm{MgO} 0,52 \%$.

PALABRAS-CLAVES: relaves, recuperación, concentrado de magnetita.

\section{MAGNETIC CONCENTRATION APPLIED TO COPPER TAILINGS}

\begin{abstract}
This work aims to develop a magnetic concentration circuit of low intensity to treat tailings produced in the treatment of copper ores. For this study were collected samples from 27 pits constructed and distributed along the crown of tailings from Manuel Antonio Matta Plant of the National Mining Company. The mineralogical analysis indicated the presence of quartz, orthoclase, magnetite, actinolite, biotite, ferrous clinochlore, calcium albite, calcite, hematite, pyrite, pyrrhotite and chalcopyrite. Chemical analysis showed a total iron content of $18.5 \%$ and $15.2 \%$ magnetic iron. Tests in a
\end{abstract}

Davis Tube indicate that a concentrate can be obtained grading $66.4 \%$ iron, $3.9 \% \mathrm{SiO}_{2}, \mathrm{Al}_{2} \mathrm{O}_{3} 0.70 \%, 0.69 \% \mathrm{MgO}$, $\mathrm{CaO} 0.60 \%$ and 0.178 sulfur, iron recovery being $79.2 \%$. Concentration tests were performed at laboratory scale in a WLIMS magnetic separator. The rougher concentrate from magnetic separator was reground to a P80 particle size which is $50 \mu \mathrm{m}$, then following cleaning tests, which gave a final product with a grade of $63.1 \%$ iron, $61.83 \%$ magnetic iron, sulfur $0.23 \%, 7 \% \mathrm{SiO}_{2}, \mathrm{Al}_{2} \mathrm{O}_{3}$ $1.16 \%, 0.52 \% \mathrm{CaO}$ and $\mathrm{MgO} 0.52 \%$.

KEYWORDS: tailings, recovery, magnetite concentrate. 


\section{INTRODUCCIÓN}

El creciente uso de metales en la industria conlleva a un paulatino agotamiento y disminución de las leyes de los minerales. Esto hace que se despierte el interés en fuentes alternativas- indicados por Díaz, (2003) y Vogt, et al, (2003)- como minerales de baja ley, minerales polimetálicos, desechos industriales, relaves etc.

Debido al alto precio de los minerales de hierro en el mercado internacional (US\$ 114,58 por tonelada métrica) puede ser una buena alternativa reprocesar los relaves de cobre para obtener concentrados de hierro (http://www.statista.com).

Según SERNAGEOMIN en Chile existen 449 tranques de relaves, de los cuales 125 están actualmente activos. En la Región de Atacama se encuentran 117 de estos tranques, que corresponden al $26,1 \%$ a nivel nacional. De estos, 45 tranques se encuentran actualmente activos y 72 no activos. Unos de los tranques activos se encuentra en la Planta Manuel Antonio Matta que tiene una masa acumulada de relave de 24.000 .000 de toneladas (www.sernageomin.cl).

El manejo de estos tranques de relaves es una operación clave en la recuperación de agua, siendo su almacenamiento la única opción, razón por la cual se debe evitar filtraciones hacia el suelo y napas subterráneas. Para obtener una tonelada de concentrado se generan casi 30 toneladas de relaves. Dado que el costo de manejar este material es alto, las compañías mineras intentan localizar los tranques o embalse de relave lo más cerca posible a la planta de procesamiento de minerales, minimizando los costos de transporte y reutilizando el agua contenida.

Un mineral de hierro puede ser dividido en tres grupos de partículas, según su respuesta frente a campos magnéticos: Partículas que manifiestan susceptibilidad magnética en campos magnéticos de baja intensidad (menor 2.000 Gauss), partículas que manifiestan susceptibilidad magnética en campos magnéticos de mediana intensidad (2.000 a 9.000 Gauss) y partículas que manifiestan susceptibilidad magnética en campos magnéticos de alta intensidad (mayor que 9.000 Gauss). Queiros y Brandão (2009) indican que la manera en que las partículas minerales se encuentran asociadas a minerales de ganga, estas influye en el comportamiento frente a campos magnéticos diferentes.

El material depositado en los tranques de relave posee un alto potencial de aprovechamiento del hierro contenido, cuyas leyes varían de $10 \%$ a $60 \%$, dependiendo del tipo de mineral tratado y de la tecnología de tratamiento de los minerales (Vieira, 2008). Un problema de las operaciones tradicionales de concentración se encuentra en la dificultad tecnológica de recuperar el hierro en las fracciones granulométricas inferiores a $20 \mu \mathrm{m}$ (Valderrama, 2011).

Este estudio tiene como objetivo recuperar el hierro magnético contenido en los relaves producidos en el tratamiento de minerales de cobre de la Planta Manuel Antonio Matta, de la Empresa Nacional de Minería, mediante diferentes etapas concentración magnética de baja intensidad. 


\section{MATERIALES Y METODOS}

Para la realización de este trabajo fueron construidas 36 calicatas distribuidas a lo largo de la corona del tranque de relaves de la Planta Manuel Antonio Matta de la Empresa Nacional de Minería. De cada calicata se sacaron muestras que fueron mezcladas, homogenizadas y cuarteadas, secadas y disgregadas hasta obtener muestras para análisis granulométrico, peso específico, análisis químico, pruebas en tubo Davis y pruebas a escala de laboratorio.

Los análisis granulométricos para determinar la distribución granulométrica de las diferentes muestras fueron realizados en húmedo, en una batería de tamices desde la malla 40 hasta la malla 400 (serie ASTM).

La determinación del peso específico se realizó por el método Le Chatelier, el que consiste en establecer la relación entre una masa y el volumen de líquido que dicha masa desplaza en el matraz.

Los análisis químicos para determinar el hierro total y hierro magnético en las muestras fueron realizadas por el método del dicromato de potasio.

El análisis mineralógico fue realizado mediante análisis por fluorescencia de rayos $\mathrm{X}$ en un espectrómetro Siemens SRS 300, y análisis por difracción de rayos $X$ en un difractómetro Siemens D 5000. También se realizaron análisis en un microscopio óptico, para identificar y cuantificar los minerales contenidos en el relave.

La determinación del índice de Bond fue realizada utilizado un molino de bola estándar de 12 " de diámetro x 12 " de largo que gira a $70 \mathrm{rpm}$, con un peso de 20.125 gramos con una área superficial total de 842 pulgadas $^{2}$. Para la remolienda se utilizó la malla $325(44,0 \mu \mathrm{m})$.

Las pruebas para concentrar las partículas magnéticas fueron realizadas en un equipo de laboratorio llamado tubo Davis. Este consiste en un electroimán que genera la intensidad de campo magnético, un tubo de separación de vidrio y un mecanismo de agitación accionado por motor. El tubo se coloca entre los polos del imán en un ángulo de aproximadamente 45 grados. El tubo se mueve hacia adelante y hacia atrás mientras gira simultáneamente. Cualquier partícula magnética presentes en la muestra en el interior del tubo se recogen en la zona de intenso magnetismo. Todas las partículas no magnéticas se descargan desde el tubo. El concentrado magnético limpio es descargado, secado y pesado para determinar el porcentaje.

Las pruebas a escala de laboratorio se llevaron a cabo en un separador magnético de tambor en húmedo de baja intensidad (WLIMS WD 20). Las condiciones usadas en esta prueba fueron: agua de mar, densidad flujo magnético 750 Gauss, porcentaje de sólidos 38\%, velocidad del tambor $50 \mathrm{rpm}$.

El concentrado rougher obtenido fue remolido a 78,3 \% bajo la malla 325 en un molino de bolas. Estas pruebas de molienda se efectuaron con el propósito de determinar el tiempo requerido para que el concentrado rougher alcanzara una granulometría de $P_{80} 50$ micrómetros, pasando posteriormente a la etapa de concentración magnética en el separador magnético de tambor en húmedo de baja intensidad. 


\section{RESULTADOS Y DISCUSIONES}

La figura 1 muestra el lugar en el tranque de relaves donde fueron construidas las calicatas, cuyo perímetro es de 2.200 metros, una altura de 27 metros y una corona de 8 metros.

Las calicatas se construyeron y distribuyeron a lo largo de la corona del tranque mediante una retroexcavadora, donde cada calicata alcanzó las dimensiones de 0,5 x 0,5 × 5 metros. El peso de relave extraído de cada calicata fue de aproximadamente 27 toneladas.

En la tabla 1 se presentan los resultados de los análisis químicos de hierro total y hierro magnético realizados a muestras de cada calicata tomada del tranque de relave.

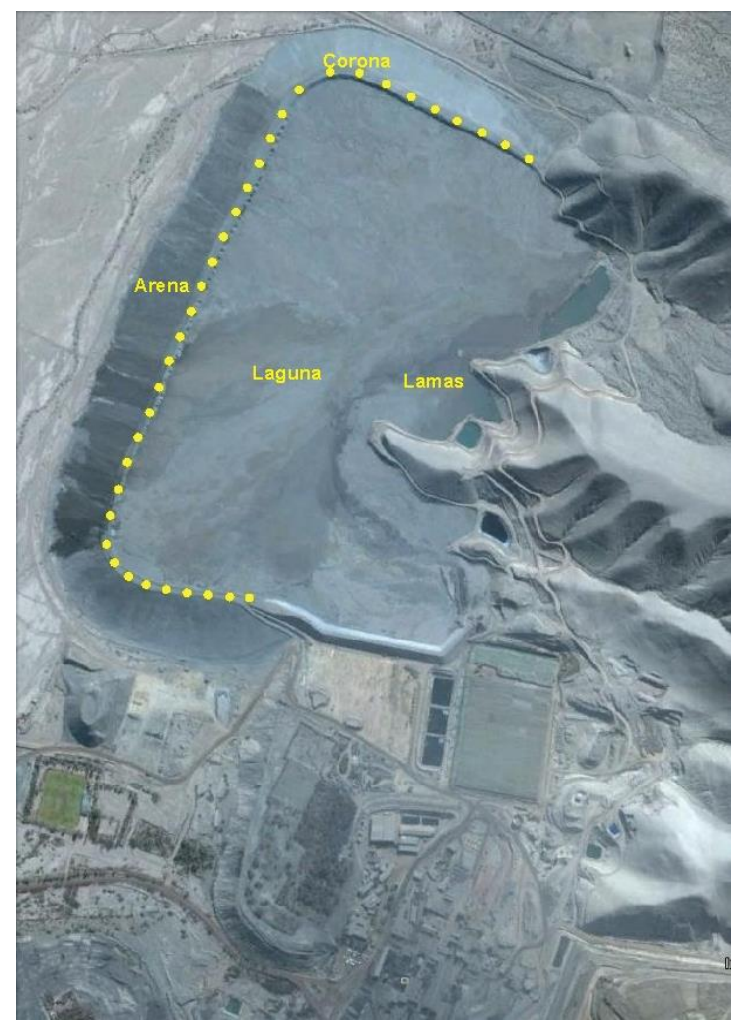

Figura 1. Tranque de relave de planta Matta indicando donde fueron construidas las calicatas

Tabla 1. Análisis químico de hierro total y magnético de cada calicata

\begin{tabular}{lllllllll}
\hline Muestra & $\mathrm{Fe}_{\mathrm{T}}$ & $\mathrm{Fe}_{\mathrm{mag}} \cdot$ & Muestra & $\mathrm{Fe}_{\mathrm{T}}$ & $\mathrm{Fe}_{\mathrm{mag}} \cdot$ & Muestra & $\mathrm{Fe}_{\mathrm{T}}$ & $\mathrm{Fe}_{\mathrm{mag} .}$ \\
$\mathrm{C}-1$ & 17,10 & 13,55 & $\mathrm{C}-10$ & 22,62 & 19,31 & $\mathrm{C}-19$ & 18,55 & 13,72 \\
$\mathrm{C}-2$ & 18,05 & 13,91 & $\mathrm{C}-11$ & 17,76 & 14,20 & $\mathrm{C}-20$ & 17,45 & 12,86 \\
$\mathrm{C}-3$ & 19,76 & 15,03 & $\mathrm{C}-12$ & 20,25 & 16,56 & $\mathrm{C}-21$ & 17,27 & 12,22 \\
$\mathrm{C}-4$ & 18,44 & 14,32 & $\mathrm{C}-13$ & 19,1 & 14,48 & $\mathrm{C}-22$ & 17,62 & 13,11 \\
$\mathrm{C}-5$ & 19,20 & 15,00 & $\mathrm{C}-14$ & 15,63 & 11,90 & $\mathrm{C}-23$ & 17,62 & 14,03 \\
$\mathrm{C}-6$ & 15,58 & 11,33 & $\mathrm{C}-15$ & 19,36 & 15,69 & $\mathrm{C}-24$ & 20,27 & 16,32 \\
$\mathrm{C}-7$ & 15,77 & 11,82 & $\mathrm{C}-16$ & 19,0 & 15,85 & $\mathrm{C}-25$ & 16,21 & 11,06 \\
$\mathrm{C}-8$ & 15,77 & 11,61 & $\mathrm{C}-17$ & 18,83 & 14,66 & $\mathrm{C}-26$ & 14,45 & 10,68 \\
$\mathrm{C}-9$ & 18,05 & 14,61 & $\mathrm{C}-18$ & 17,45 & 13,57 & $\mathrm{C}-27$ & 16,74 & 12,89 \\
\hline
\end{tabular}


Se observa que las muestras de relaves presentan leyes de hierro total que fluctúan entre $14,45 \%$ y $22,62 \%$ y de hierro magnético entre $10,68 \%$ y $19,31 \%$.

Las muestras de cada calicata fueron molidas para que el material tenga un tamaño granulométrico entre un 80 y 85 \% bajo la malla 325 Tyler, que es la granulometría requerida para realizar el ensayo en el tubo Davis.

La tabla 2 muestra los resultados de los análisis químicos de cada calicata obtenida en el tubo Davis, observándose que las leyes de hierro en el concentrado obtenido son elevadas, siendo la menor ley de hierro de $62,41 \%$, para la calicata C-8 y la de mayor ley de hierro 67,86 \% para la calicata C-17.

La muestra de las 27 calicatas fueron mezcladas con el objetivo de tener una muestra representativa del tranque de relave. Esta muestra fue homogenizada, cuarteada, secada y disgregada hasta obtener muestras para análisis granulométrico, químico, peso específico y pruebas de concentración magnética.

Tabla 2. Resultados de análisis químico para cada calicata en el tubo Davis

\begin{tabular}{|c|c|c|c|c|c|c|c|}
\hline Muestra & $\begin{array}{l}\text { \%Fe } \\
\text { D.T.T }\end{array}$ & $\% \mathrm{SiO}_{2}$ D.T.T & \%S D.T.T & Muestra & \%Fe D.T.T & $\begin{array}{l}\% \mathrm{SiO}_{2} \\
\text { D.T.T }\end{array}$ & \%S D.T.T \\
\hline$C-1$ & 65,14 & 4,70 & 0,140 & $C-15$ & 65,37 & 3.90 & 0,164 \\
\hline$C-2$ & 63,51 & 5,20 & 0,143 & $C-16$ & 67,14 & 4.40 & 0,289 \\
\hline$C-3$ & 64,78 & 4,60 & 0,314 & $C-17$ & 67,86 & 3.50 & 0,157 \\
\hline C-4 & 66,59 & 3,60 & 0,320 & $C-18$ & 65,55 & 5.16 & 0,159 \\
\hline C-5 & 65,50 & 4,50 & 0,213 & C-19 & 66,26 & 5.60 & 0,330 \\
\hline C-6 & 62,96 & 5,80 & 0,219 & $C-20$ & 66,61 & 4.32 & 0,308 \\
\hline$C-7$ & 64,96 & 5,20 & 0,231 & $C-21$ & 64,67 & 5.92 & 0,289 \\
\hline C-8 & 62,41 & 6,80 & 0,196 & $C-22$ & 65,55 & 4.80 & 0,218 \\
\hline C-9 & 66,41 & 3,90 & 0,178 & $C-23$ & 67,15 & 4.44 & 0,203 \\
\hline C-10 & 67,50 & 3,10 & 0,100 & $C-24$ & 66,88 & 4.64 & 0,168 \\
\hline$C-11$ & 66,97 & 4,30 & 0,114 & $C-25$ & 66,61 & 4.68 & 0,150 \\
\hline$C-12$ & 66,52 & 4,80 & 0,128 & $C-26$ & 67,15 & 3.96 & 0,158 \\
\hline$C-13$ & 66,43 & 4,70 & 0,173 & $C-27$ & 67,50 & 3.72 & 0,192 \\
\hline C-14 & 62,97 & 4,40 & 0,213 & & & & \\
\hline
\end{tabular}

Se puede observar en la tabla que al someter la muestra de cada calicata a concentración magnética en el tubo Davis, las leyes de hierro en el concentrado obtenido son bastantes altas (entre $62,41 \% \mathrm{Fe}$ y $67,86 \% \mathrm{Fe}$ ). Las leyes de $\mathrm{SiO}_{2}$ y azufre en el concentrado magnético obtenido se encuentran sobre las especificaciones requeridas.

Los resultados de los análisis granulométricos se muestran en la Tabla 3. Se puede observar que el relave tiene un tamaño de $100 \%$ menor a 10 mallas, que el 55,43\% de las partículas tiene un tamaño menor a 105 micrómetros y que el 19,85\% de las partículas tiene un tamaño menor a 44 micrómetros.

En análisis mineralógico indicó la presencia de cuarzo, ortoclasa, magnetita, actinolita, biotita, albita cálcica, calcita, hematita, pirita, pirrotina y calcopirita. 
Tabla 3. Análisis granulométrico del relave de cobre

\begin{tabular}{cccccc}
\hline Malla & $\begin{array}{c}\text { Abertura } \\
(\mathrm{mm})\end{array}$ & $\begin{array}{c}\text { Peso } \\
(\mathrm{g})\end{array}$ & $\begin{array}{c}\text { Peso } \\
(\%)\end{array}$ & $\begin{array}{c}\text { Retenido } \\
(\%)\end{array}$ & $\begin{array}{c}\text { Pasante } \\
(\%)\end{array}$ \\
ASTM & 0,595 & 0,34 & 0,12 & 0,15 & 99,87 \\
30 & 0,420 & 0,23 & 0,09 & 0,22 & 99,78 \\
40 & 0,297 & 10,17 & 3,99 & 4,21 & 95,79 \\
50 & 0,210 & 20,16 & 7,90 & 12,11 & 87,89 \\
70 & 0,149 & 35,61 & 13,96 & 26,07 & 73,93 \\
100 & 0,105 & 47,20 & 18,50 & 44,57 & 55,43 \\
140 & 0,074 & 44,63 & 17,50 & 62,07 & 37,93 \\
200 & 0,053 & 33,34 & 13,07 & 75,14 & 24,86 \\
270 & 0,044 & 12,79 & 5,01 & 80,15 & 19,85 \\
325 & 0,037 & 6,650 & 2,61 & 82,76 & 17,24 \\
400 & $-0,037$ & 43,99 & 17,24 & 100 & 0 \\
-400 & & & & & \\
\hline
\end{tabular}

El peso específico del relave, determinado a través del método de Le Chatelier, fue de $3,06 \mathrm{Kg} / \mathrm{L}$.

La Tabla 4 presenta los resultados de los análisis químicos de hierro total y hierro magnético realizados a la muestra de relave de cobre. Se observa que la ley de hierro total de $19,31 \%$, hierro magnético $14,52 \%$ y Fe Dtt $66,61 \%$.

Tabla 4. Análisis de químico del compósito de relave

\begin{tabular}{ccccc}
\hline Muestra & $\mathrm{Fe}_{\mathrm{T}}$ & $\mathrm{Dtt}$ & $\mathrm{Fe} \mathrm{Dtt}$ & $\mathrm{Fe} \mathrm{Mag}$ \\
& $(\%)$ & $(\%)$ & $(\%)$ & $(\%)$ \\
Relave & 19,31 & 21,8 & 66,61 & 14,52 \\
\hline
\end{tabular}

En la tabla 5 se muestran los resultados obtenidos al concentrar el relave de cobre en el separador magnético de tambor en húmedo de baja intensidad. Se puede observar en la tabla que la ley de hierro alimentada a la prueba fue de 19,31\%, obteniéndose un concentrado y un relave con $50,53 \%$ y $4,9 \%$, respectivamente. Los resultados obtenidos en esta prueba indican que la ley de hierro en el concentrado es baja, por lo cual requiere de ser sometida a etapas de limpieza para tener concentrados con leyes de hierro comerciales.

Tabla 5. Resultados de la concentración magnética rougher al relave

\begin{tabular}{lcccc}
\hline Muestra & Peso $(\mathrm{kg})$ & $\mathrm{Fe}_{\mathrm{T}}(\%)$ & $\mathrm{R}_{\mathrm{P}}(\%)$ & $\mathrm{R}_{\mathrm{M}}(\%)$ \\
Concentrado & 39,4 & 50,53 & 31,6 & 82,7 \\
Relave & 85,2 & 4,9 & 68,4 & 17,3 \\
Alimentación & 124,6 & 19,31 & 100 & 100 \\
\hline
\end{tabular}

El concentrado rougher presentó una granulometría de $56,4 \%$ menor a 200 mallas (74 $\mu \mathrm{m})$. Este concentrado fue remolido hasta una granulometría de las partículas de $78,3 \%$ menor a 325 mallas $(44 \mu \mathrm{m})$. El peso específico del concentrado rougher fue de $4,24(\mathrm{Kg} / \mathrm{L})$. La determinación del Índice de Bond (Wi) al concentrado rougher, realizada en duplicado, fue $32,7 \mathrm{kWh} / \mathrm{t}$ métrica.

El concentrado remolido se sometió a pruebas de separación magnética finisher con la finalidad de incrementar la ley de hierro. En estas pruebas se realizaron con agua de mar, con un porcentaje de sólidos de 35\%. En la tabla 6 se presentan los resultados de los productos obtenidos en las tres etapas finisher. 
Se observa que la ley de hierro en el concentrado de la primera concentración finisher se incrementa a 58,7 \% con una recuperación metalúrgica de 97,0\%. En el segunda etapa de limpieza la ley de hierro en el concentrado se incrementa a 62,0 \% mientras que la recuperación alcanza el 99,4\%. La ley de hierro en la tercera concentración finisher se incrementa a $63,1 \%$ con una recuperación de $99,5 \%$, siendo el peso específico del concentrado ( $3^{\mathrm{er}}$ Finisher) de $4,76(\mathrm{Kg} / \mathrm{L})$.

Tabla 6. Resultados obtenidos en las pruebas realizada al concentrado rougher

\begin{tabular}{l|c|c|c|c|c|c|c|c|c|}
\hline \multirow{2}{*}{ Muestra } & \multicolumn{3}{|c|}{$1^{\circ}$ Finisher } & \multicolumn{3}{c|}{$2^{\circ}$ Finisher } & \multicolumn{3}{c|}{$3^{\circ}$ Finisher } \\
\cline { 2 - 10 } & $\begin{array}{c}\text { Peso } \\
(\mathrm{kg})\end{array}$ & $\begin{array}{c}\mathrm{Fe} \\
(\%)\end{array}$ & $\begin{array}{c}\mathrm{R}_{\mathrm{M}} \\
(\%)\end{array}$ & $\begin{array}{c}\text { Peso } \\
(\mathrm{kg})\end{array}$ & $\begin{array}{c}\mathrm{Fe} \\
(\%)\end{array}$ & $\begin{array}{c}\mathrm{R}_{\mathrm{M}} \\
(\%)\end{array}$ & $\begin{array}{c}\text { Peso } \\
(\mathrm{kg})\end{array}$ & $\begin{array}{c}\mathrm{Fe}_{\mathrm{T}} \\
(\%)\end{array}$ & $\begin{array}{c}\mathrm{R}_{\mathrm{M}} \\
(\%)\end{array}$ \\
\hline Concentrado & 32,9 & 58,7 & 97,0 & 31,0 & 62,0 & 99,4 & 30,3 & 63,1 & 99,5 \\
\hline Relave & 6,5 & 9,3 & 3,0 & 1,9 & 11,6 & 0,6 & 0,7 & 14,9 & 0,5 \\
\hline Alimentación & 39,4 & 50,5 & 100,0 & 32,9 & 58,7 & 100,0 & 31,0 & 62,0 & 100,0 \\
\hline
\end{tabular}

A pesar de obtener un concentrado con ley de hierro comercial, este requiere aún de etapas complementarias de flotación para disminuir el contenido de impurezas se encuentra fuera de las especificaciones de mercado $\left(\mathrm{SiO}_{2}\right.$ y azufre). Las pruebas de flotación están siendo realizadas, para obtener un concentrado final que cumpla con niveles de impurezas adecuadas.

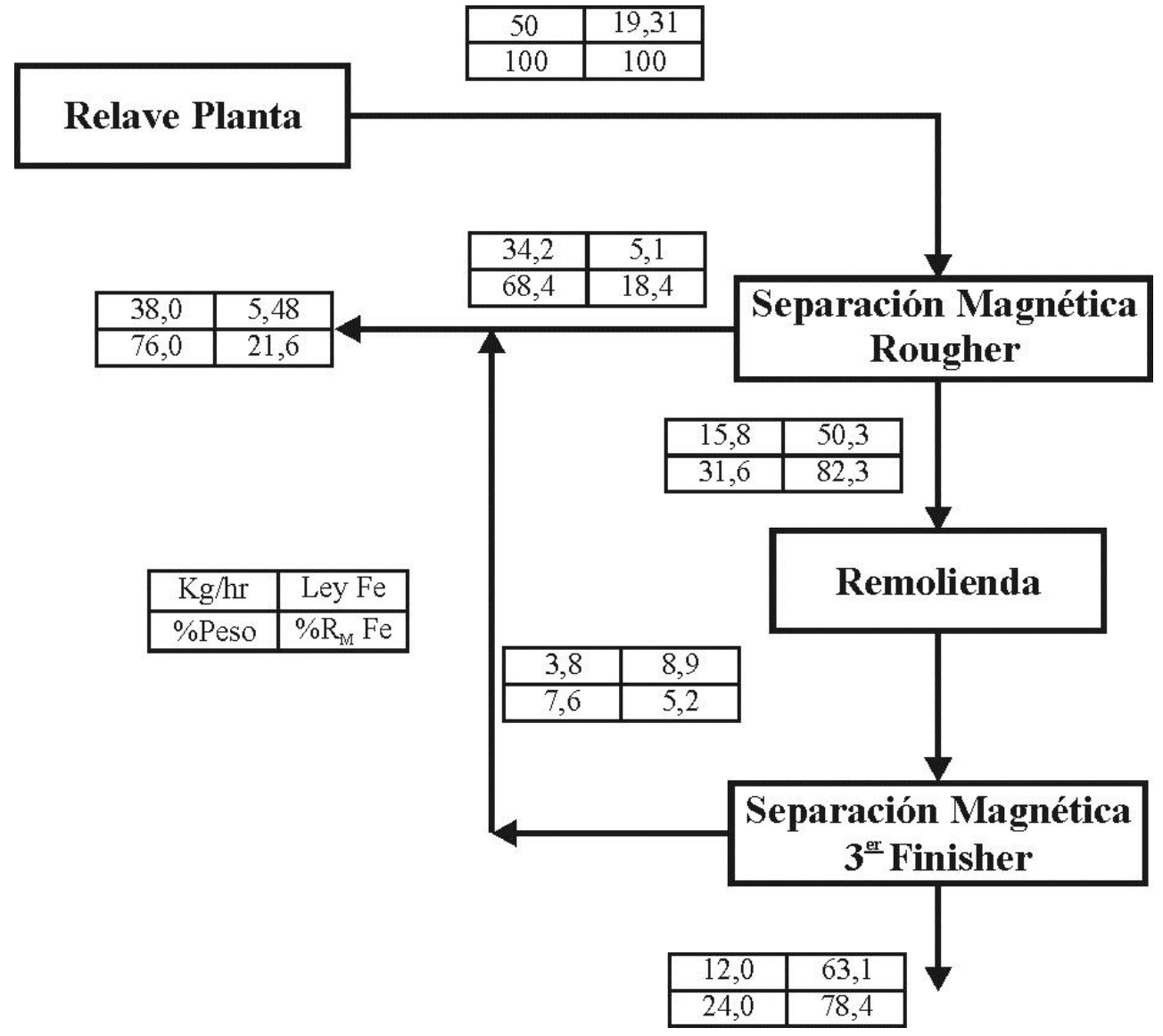

Figura 2. Diagrama de flujo para tratar el relave de planta Matta

La figura 2 presenta el diagrama de flujo con los análisis de hierro, porcentaje de masa en peso y la recuperación metalúrgica propuesto en este trabajo. En este diagrama se propone 
una etapa rougher, remolienda del concentrado para posteriormente someter el concentrado a tres etapas de limpieza.

Se puede observar que es posible obtener un concentrado final con una ley de hierro de $63,1 \%$, una recuperación en peso de $24 \%$ y una recuperación metalúrgica de $78,4 \%$.

\section{CONCLUSIONES}

Los resultados de este estudio indican que es posible un mayor aprovechamiento de la masa $(24,0 \%)$ de los relaves que actualmente se encuentran depositados en el tranque de relaves de cobre. Ellos indican que es posible recuperar el hierro magnético, como una alternativa técnicamente factible. El concentrado de hierro producido a través del tratamiento del relave presenta una calidad química dentro de las especificaciones requeridas.

Este proceso para recuperar minerales contenidos en los relaves entrega una adecuada recuperación global, lo que indica que cerca de 5.760 .000 de toneladas de relave dejarían de ser depositadas en el tranque. Este nuevo circuito necesita flexibilizar la operación con tal de aumentar la capacidad de concentración de materiales contenidos en los relaves.

\section{BIBLIOGRAFIA}

1. Díaz, D. Proyecto de Recuperación de Hierro desde Relaves de las Etapas de Concentración Magnética en Planta de Pellet. Trabajo de Titulación. Departamento de Metalurgia, Universidad de Atacama 2003.

2. Vogt, M.L, Dold B., Spangenberg, J.E.,. Mineralogical and geochemical characterization of the active tailings impoundment Talabre, Chuquicamata, Chile. X Congreso Geológico Chileno, Concepción. 2003.

3. http://www.statista.com/statistics/300419/monthly-iron-ore-prices/

4. www.sernageomin.cl/pdf/mineria/seguridad/estudios/catastro_depositos_de_relaves_201 0 .

5. Queiroz, L. A. y Brandão, P. R. G. Aspectos Mineralógicos Relacionados à Concentração Magnética de Minério de Ferro Itabirítico. Metalurgia \&Materiais, Volume 65, p. 148-151, abr. 2009.

6. Vieira, M.G. Produção de um pellet feed a partir da concentração do rejeito da flotaçãomecânica da Samarco. Dissertação de Mestrado, Curso de PósGraduaçãoemEngenharia Metalúrgica e de Minas, UFMG, 90 p., 2008.

7. Valderrama, L., Santander, M., Díaz, D., Meléndez, M. Recuperación de hierro de relaves de la concentración magnética. XXIV ENTMME. Pág. 694-701. Salvador/Bahía 2011. 